\title{
A Class of Dilation Integral Equations
}

\author{
J. C. S. de Miranda (Corresponding author) \\ Institute of Mathematics and Statistics, University of São Paulo \\ São Paulo 05508-090, Brazil \\ E-mail: simon@ime.usp.br
}

A. P. Franco Filho

Institute of Mathematics and Statistics, University of São Paulo

São Paulo 05508-090, Brazil

E-mail: apadua@ime.usp.br

Received: March 26, 2012 Accepted: April 10, 2012 Online Published: May 28, 2012

doi:10.5539/jmr.v4n3p1 URL: http://dx.doi.org/10.5539/jmr.v4n3p1

This work was partially supported by FAPESP grant 2008/51097-6

\begin{abstract}
We present a class of dilation integral equations. The equations in this class depend on a dilation parameter $a \in \mathbb{R}$. The existence of non trivial solutions in $L^{1}(\mathbb{R})$ is studied as a function of the dilation parameter. The main result establishes the non existence of these solutions for $|a|<1$, a necessary and sufficient condition for the existence of solutions with non vanishing integrals in case $|a|>1$, and sufficient conditions for these equations to have no solutions but the trivial one or to have an infinitude of non trivial solutions in case $|a|=1$. In all these cases, the dimension of the space of $L^{1}(\mathbb{R})$-solutions is determined. When $|a|>1$ we have succeeded in writing the frequency domain representation of the solutions as convergent infinite products.
\end{abstract}

Keywords: Convolution, Dilation integral equation, Existence of solutions, Fourier transform, Uniqueness of solution

\section{Introduction}

A functional equation is called a dilation equation when the unknown function $f$ is calculated at least at the arguments $x$ and $a x, a \neq 1$, and these values simultaneously appear in this equation. In general these equations are not easy to solve. In this work we present a class of integral equations with dilation. They are of convolution type and this permits the use of Fourier transform methods to successfully study them. The transformed equations are simple, i.e., are neither differential nor integral, dilation equations. See Strang (1989), Heil (1994) and references therein for an introduction to this type of equations.

Let $g: \mathbb{R} \rightarrow \mathbb{R}$, be an integrable function, i.e., $g \in L^{1}(\mathbb{R})$. We will be interested in the following class of integral equations:

$$
f(x)=\int_{\mathbb{R}} f(a(x-z)) g(z) \mathrm{d} z
$$

or, in shorter notation,

$$
f=f(a \cdot) * g
$$

where $f(a \cdot)(x)=f(a x)$, and $*$ is the convolution product.

In this work we will be interested in $L^{1}(\mathbb{R})$ solutions only. We will use the following notations:

The Fourier transform of a function $f \in L^{1}(\mathbb{R})$, will be denoted by $\hat{f}$. The Fourier transform is denoted by $\mathcal{F}$. We use the following definition of this transform:

$$
f \rightarrow \mathcal{F}(f)=\hat{f} \quad \hat{f}(\omega)=\int_{\mathbb{R}} f(x) e^{-\mathrm{i} \omega x} \mathrm{~d} x
$$

This article is organized as follows: In section two we state and prove the main result and in section three we finish this work with some final remarks. 


\section{Main Results}

Let us denote by $\mathbb{S}$ the set of solutions of the dilation integral equation (1). Clearly, $\mathbb{S} \neq \emptyset$, since $f=0$ is a solution of (1) whatever the value of $a$ is. From here on we will refer to this solution as the trivial one.

Proposition 1 The integral equation (1) is linear, i.e., if $f_{1}$ and $f_{2}$ are solutions to (1) so is $\alpha f_{1}+\beta f_{2}$ for all $\alpha$ and $\beta$ in $\mathbb{R}$.

Proof. $\alpha f_{1}+\beta f_{2}=\alpha f_{1}(a \cdot) * g+\beta f_{2}(a \cdot) * g=\left(\alpha f_{1}(a \cdot)+\beta f_{2}(a \cdot)\right) * g=\left(\left(\alpha f_{1}+\beta f_{2}\right)(a \cdot)\right) * g$

Thus, $\mathbb{S}$ is a vector space.

Proposition 2 If $\|g\|_{1}<|a|$ then the only solution to (1) is the trivial solution $f=0 \in L^{1}(\mathbb{R})$.

Proof. Suppose $f$ is a solution and remember that $\left(L^{1}(\mathbb{R}),+, \cdot, *\right)$ is a Banach Algebra.

Thus

$$
\|f\|_{1}=\|f(a \cdot) * g\|_{1} \leq\|f(a \cdot)\|_{1}\|g\|_{1}=\frac{1}{|a|}\|f\|_{1}\|g\|_{1}
$$

If $\|f\|_{1} \neq 0$ then

$$
\|f\|_{1}<\frac{1}{|a|}\|f\|_{1}|a|=\|f\|_{1},
$$

a contradiction. Thus $f=0$ is the only solution.

Lemma 1 If $f$ and $g$ are in $L^{1}(\mathbb{R})$, and $f \geq 0$ and $g \geq 0$ then $\|f * g\|_{1}=\|f\|_{1}\|g\|_{1}$.

Proof. $\|f * g\|_{1}=\int_{\mathbb{R}}\left|\int_{\mathbb{R}} f(x-z) g(z) \mathrm{d} z\right| \mathrm{d} x=\int_{\mathbb{R}} \int_{\mathbb{R}} f(x-z) g(z) \mathrm{d} z \mathrm{~d} x=\int_{\mathbb{R}} \int_{\mathbb{R}} f(w) g(z) \mathrm{d} z \mathrm{~d} w=\|f\|_{1}\|g\|_{1}$.

Proposition 3 Suppose that $g \geq 0$ and that equation (1) admits a non trivial non negative solution $f$. Then $\|g\|_{1}=|a|$.

Proof. Using Lemma 1 we can write

$$
0 \neq\|f\|_{1}=\|f(a \cdot) * g\|_{1}=\|f(a \cdot)\|_{1}\|g\|_{1}=\frac{1}{|a|}\|f\|_{1}\|g\|_{1}
$$

from which

$$
\frac{1}{|a|}\|g\|_{1}=1
$$

Proposition 4 If $f$ is a solution of equation (1) then its Fourier transform obeys the functional equation

$$
\hat{f}(\omega)=\hat{f}\left(\frac{\omega}{a}\right) \frac{\hat{g}(\omega)}{|a|} .
$$

Proof. Taking the Fourier transform of (1) and using the convolution theorem and the scaling property of the Fourier transform, we have

$$
\hat{f}(\omega)=\widehat{f(a \cdot)}(\omega) \hat{g}(\omega)=\frac{1}{|a|} \hat{f}\left(\frac{\omega}{a}\right) \hat{g}(\omega) .
$$

Proposition 5 If $f$ is a solution of equation (1) with $|a|>1$ such that $\hat{f}(0) \neq 0$ then its Fourier transform is given by

$$
\hat{f}(\omega)=\hat{f}(0) \prod_{n=0}^{\infty}\left(\hat{g}\left(\frac{\omega}{a^{n}}\right)|| a \mid\right)=\int_{\mathbb{R}} f(x) \mathrm{d} x \prod_{n=0}^{\infty}\left(\hat{g}\left(\frac{\omega}{a^{n}}\right) /|a|\right) .
$$

Proof. Iteration of relation (4) yields

$$
\forall m \in \mathbb{N} \quad \hat{f}(\omega)=\hat{f}\left(\frac{\omega}{a^{m+1}}\right) \prod_{n=0}^{m}\left(\hat{g}\left(\frac{\omega}{a^{n}}\right)|| a \mid\right) .
$$

Since $f \in L^{1}(\mathbb{R})$, its Fourier transform is continuous. See (Pinsky, 2009). Thus, for $|a|>1$, we have for all $\omega \in \mathbb{R}$,

$$
\lim _{m \rightarrow \infty} \hat{f}\left(\frac{\omega}{a^{m+1}}\right)=\hat{f}\left(\lim _{m \rightarrow \infty} \frac{\omega}{a^{m+1}}\right)=\hat{f}(0) \neq 0 .
$$


Thus, for all $\omega$ there is an $m_{0}$ such that for all $m>m_{0}$ we have $\hat{f}\left(\frac{\omega}{a^{m+1}}\right) \neq 0$ so that

$$
\prod_{n=0}^{m}\left(\hat{g}\left(\frac{\omega}{a^{n}}\right) /|a|\right)=\frac{\hat{f}(\omega)}{\hat{f}\left(\frac{\omega}{a^{m+1}}\right)}
$$

and, taking limits, we have

$$
\lim _{m \rightarrow \infty} \prod_{n=0}^{m}\left(\hat{g}\left(\frac{\omega}{a^{n}}\right) /|a|\right)=\lim _{m \rightarrow \infty} \frac{\hat{f}(\omega)}{\hat{f}\left(\frac{\omega}{a^{m+1}}\right)}=\frac{\hat{f}(\omega)}{\hat{f}(0)}
$$

which guarantees the existence of the infinite product $\prod_{n=0}^{\infty}\left(\hat{g}\left(\frac{\omega}{a^{n}}\right) /|a|\right)$. Thus,

$$
\hat{f}(\omega)=\lim _{m \rightarrow \infty}\left(\hat{f}\left(\frac{\omega}{a^{m+1}}\right) \prod_{n=0}^{m}\left(\hat{g}\left(\frac{\omega}{a^{n}}\right) /|a|\right)\right)=\hat{f}(0) \prod_{n=0}^{\infty}\left(\hat{g}\left(\frac{\omega}{a^{n}}\right) /|a|\right) .
$$

Finally,

$$
\hat{f}(0)=\int_{\mathbb{R}} f(x) e^{\mathrm{i} 0 x} \mathrm{~d} x=\int_{\mathbb{R}} f(x) \mathrm{d} x .
$$

This completes the proof.

Proposition 6 If equation (1) with $|a|>1$ admits a solution $f$ such that $\int_{\mathbb{R}} f(x) \mathrm{d} x \neq 0$ then $\int_{\mathbb{R}} g(x) \mathrm{d} x=|a|$.

Proof. Under these conditions, equation (8) guarantees the existence of the limit

$$
\lim _{m \rightarrow \infty} \prod_{n=0}^{m}\left(\hat{g}\left(\frac{\omega}{a^{n}}\right) /|a|\right) .
$$

Taking $\omega=0$ in equation (8), we have $\prod_{n=0}^{\infty}(\hat{g}(0) /|a|)=1$ which directly implies $\hat{g}(0)=|a|$. Now, from (3), $\hat{g}(0)=\int_{\mathbb{R}} g(x) e^{0} \mathrm{~d} x$. This completes this proof.

Let us denote by $\mathbb{V}_{0}$ the vector subspace of $L^{1}(\mathbb{R})$ defined by $\mathbb{V}_{0}=\left\{f \in L^{1}(\mathbb{R}): \int_{\mathbb{R}} f(x) \mathrm{d} x=0\right\}$. Observe that we have $\mathbb{V}_{0}=\left\{f \in L^{1}(\mathbb{R}): \hat{f}(0)=0\right\}$. From here on we will also write $\mathbb{W}=\left\{f \in \mathbb{R}^{\mathbb{R}}: f(0)=0\right\}$, and $\mathbb{Y}=L^{1}(\mathbb{R}) \backslash \mathbb{V}_{0}$.

Proposition 7 If equation (1) with $|a|>1$ admits a solution in $\mathbb{Y}$ and $g \geq 0$ then $\|g\|_{1}=|a|$.

Proof. Immediate.

Proposition 8 Consider equation (1) with $|a|>1$. If the infinite product $\prod_{n=0}^{\infty}\left(\hat{g}\left(\frac{\omega}{a^{n}}\right) /|a|\right)$ converges to a limit function whose value at $\omega=0$ is different from zero and which is Fourier invertible, i.e., if

$$
\prod_{n=0}^{\infty}\left(\hat{g}\left(\frac{\omega}{a^{n}}\right) /|a|\right) \in \mathcal{F}\left[L^{1}(\mathbb{R})\right] \backslash \mathbb{W},
$$

then equation (1) admits non trivial solutions. All the solutions to (1) are given by

$$
f=k \mathcal{F}^{-1}\left(\prod_{n=0}^{\infty}\left(\hat{g}\left(\frac{\omega}{a^{n}}\right) /|a|\right)\right) .
$$

for all $k \in \mathbb{R}$.

Proof. It suffices to show that equation (10) generates a solution for $k=1$, because, in this case, equation (1) will have a non trivial solution with $\hat{f}(0) \neq 0$ which, by proposition (5), implies that all solutions to (1) such that $\hat{f}(0) \neq 0$ are written in the form of equation (5) or equivalently of equation (10) with $k=\hat{f}(0)$. Moreover, if $\hat{f}(0)=0$ then, letting $m \rightarrow \infty$ in equation (6) we have

$$
\hat{f}(\omega)=\hat{f}(0) \prod_{n=0}^{\infty}\left(\hat{g}\left(\frac{\omega}{a^{n}}\right) /|a|\right)=0,
$$


the trivial solution that corresponds to the choice $k=0$ in (10).

Taking $k=1$, we have

$$
\mathcal{F}(f(a \cdot))=\frac{1}{|a|}(\mathcal{F}(f))\left(\frac{1}{a} \cdot\right)=\frac{1}{|a|}\left(\prod_{n=0}^{\infty}\left(\hat{g}\left(\frac{\frac{\omega}{a}}{a^{n}}\right)|| a \mid\right)\right)=\frac{1}{|a|}\left(\prod_{n=1}^{\infty}\left(\hat{g}\left(\frac{\omega}{a^{n}}\right) /|a|\right)\right)
$$

and

$f=\mathcal{F}^{-1}\left(\prod_{n=0}^{\infty}\left(\hat{g}\left(\frac{\omega}{a^{n}}\right) /|a|\right)\right)=\mathcal{F}^{-1}\left(\frac{1}{|a|} \hat{g}(\omega) \prod_{n=1}^{\infty}\left(\hat{g}\left(\frac{\omega}{a^{n}}\right) /|a|\right)\right)=\mathcal{F}^{-1}(\hat{g}(\omega)) * \mathcal{F}^{-1}\left(\frac{1}{|a|} \prod_{n=1}^{\infty}\left(\hat{g}\left(\frac{\omega}{a^{n}}\right) /|a|\right)\right)=g * f(a \cdot)$,

that is, $f$ is a solution of (1). This completes the proof.

Proposition 9 If $0<|a|<1$ then the unique solution to the dilation integral equation (1) is the trivial one, $f=0$.

Proof. From (6) we have

$$
\forall \omega \in \mathbb{R} \quad \hat{f}(\omega)=\lim _{m \rightarrow \infty} \hat{f}\left(\frac{\omega}{a^{m+1}}\right) \prod_{n=0}^{m}\left(\hat{g}\left(\frac{\omega}{a^{n}}\right) /|a|\right) .
$$

Now, since $0<|a|<1$, for all $\omega \neq 0$, we have

$$
\lim _{m \rightarrow \infty}\left|\frac{\omega}{a^{m+1}}\right|=\lim _{m \rightarrow \infty}\left|\frac{\omega}{a^{m}}\right|=\infty .
$$

Thus, by the Riemann Lebesgue Lemma we have $\lim _{m \rightarrow \infty} \hat{f}\left(\frac{\omega}{a^{m+1}}\right)=0$ as well as $\lim _{m \rightarrow \infty}\left(\hat{g}\left(\frac{\omega}{a^{m}}\right) /|a|\right)=0$. Consequently,

$$
\forall \omega \neq 0 \quad \hat{f}(\omega)=0
$$

and, since $\hat{f}$ is continuous, we also have $\hat{f}(0)=0$. This completes the proof.

Proposition 10 If $a=0$ then equation (1) (with point-wise equality) has non trivial solutions if and only if $\int_{\mathbb{R}} g(x) \mathrm{d} x=1$ and, in this case, $\mathbb{S}$ is the set of constant functions. However, these solutions are not in $L^{1}(\mathbb{R})$.

Proof. Taking $a=0$ in (1), we have

$$
f(x)=\int_{\mathbb{R}} f(0) g(z) \mathrm{d} z=f(0) \int_{\mathbb{R}} g(z) \mathrm{d} z=k f(0)
$$

Now, $f(0)=k f(0)$ and $f(0)=0$ or $k=\int_{\mathbb{R}} g(z) \mathrm{d} z=1$.

Remark 1 If in Proposition 10 we use equality a.e. with respect to the Lebesgue measure instead of point-wise equality then equation (1) has non trivial solutions if and only if $\int_{\mathbb{R}} g(x) \mathrm{d} x \neq 0$ and $\mathbb{S}$ is the set of a.e. constant functions. We observe again that these are not $L^{1}-$ solutions.

Proof. Equation (12) is still valid and we have $f(x)=k f(0)$ a.e.

Proposition 11 If $a=1$ and $\hat{g} \neq 1$ a.e. then equation (1) has no solutions but the trivial one.

Proof. From equation (4), we have $\hat{f}(\omega)=\hat{f}(\omega) \hat{g}(\omega)$. Thus

$$
\forall \omega \in \mathbb{R} \quad \hat{f}(\omega)=0 \vee \hat{g}(\omega)=1 .
$$

Under the assumption $\hat{g} \neq 1$ a.e., we have $\hat{f}(\omega)=0$ a.e., and, from the continuity of $\hat{f}$, we conclude that $\hat{f}=0$. Thus $f=0$ a.e.

Proposition 12 If $a=1$ then there exists $g \in L^{1}(\mathbb{R})$ such that equation (1) admits infinitely many linearly independent solutions.

Proof. Let $\varphi$ be a $C^{\infty}$-function such that for all $x \in[-1,1]$, we have $\varphi(x)=1$ and that, for all $x \in \mathbb{R} \backslash[-2,2]$, we have $\varphi(x)=0$. Then, $\varphi \in \mathcal{S}(\mathbb{R})$, the set of rapidly decreasing $C^{\infty}$-functions on $\mathbb{R}$. Thus, we can define $g$ by taking $\hat{g}=\varphi$, and we will have $g=\mathcal{F}^{-1} \varphi \in \mathcal{S}(\mathbb{R}) \subset L^{1}(\mathbb{R})$. (See Rudin, 1980). We will take $\varphi$ an even function in order to guarantee that $g$ is a real valued function. Now, for every even $C^{\infty}$-function, $\psi$, with support contained in $[-1,1]$, we can take $\hat{f}=\psi \in \mathcal{S}(\mathbb{R})$ and obtain the real valued function $f=\mathcal{F}^{-1} \psi \in \mathcal{S}(\mathbb{R}) \subset L^{1}(\mathbb{R})$, a solution 
of (1). Observe that we have $\hat{f}(\omega)=\hat{f}(\omega) \hat{g}(\omega)$ for all $\omega \in \mathbb{R}$, and, consequently, the fulfillment of (1). Clearly, the set of the even $C^{\infty}$-functions with support contained in $[-1,1]$ is an infinite dimensional vector subspace of $L^{1}(\mathbb{R})$. Since linear independence is preserved by $\mathcal{F}^{-1}$, the proof is complete.

Proposition 13 If $a=-1$ and $\hat{g} \cdot \hat{g}(-1 \cdot) \neq 1$ a.e. then equation (1) has no solutions but the trivial one.

Proof. From equation (4), we have $\hat{f}(\omega)=\hat{f}(-\omega) \hat{g}(\omega)$. Calculating $\hat{f}(-\omega)$ we have $\hat{f}(-\omega)=\hat{f}(\omega) \hat{g}(-\omega)$ and substituting in the previous formula we get $\hat{f}(\omega)=\hat{f}(\omega) \hat{g}(-\omega) \hat{g}(\omega)$. Thus

$$
\forall \omega \in \mathbb{R} \quad \hat{f}(\omega)=0 \vee \hat{g}(\omega) \hat{g}(-\omega)=1,
$$

and, if $\hat{g} . \hat{g}(-1 \cdot) \neq 1$ a.e., then $\hat{f}=0$ a.e. and the continuity of $\hat{f}$ implies $\hat{f}=0$. Thus, $f=0$ a.e. and the proof is complete.

Proposition 14 If $a=-1$ then there exists $g \in L^{1}(\mathbb{R})$ such that equation (1) admits infinitely many linearly independent solutions.

Proof. Analogous to the proof of Proposition 12. The same choices of $\hat{g}$ and $\psi$ are made. Since $\hat{f}=\psi$ is an even function, we have $\hat{f}(\omega)=\hat{f}(\omega) \hat{g}(\omega)=\hat{f}(-\omega) \hat{g}(\omega)$.

Now we state our main result, which organizes and joins the results of the propositions. Remember that $\mathbb{Y}=$ $L^{1}(\mathbb{R}) \backslash\left\{f \in L^{1}(\mathbb{R}): \int_{\mathbb{R}} f(x) \mathrm{d} x=0\right\}$ and that $\mathbb{W}=\left\{f \in \mathbb{R}^{\mathbb{R}}: f(0)=0\right\}$.

Theorem 1 Let $g \in L^{1}(\mathbb{R})$ and $a \in \mathbb{R}$. Consider the linear dilation integral equation

$$
f(x)=\int_{\mathbb{R}} f(a(x-z)) g(z) \mathrm{d} z .
$$

Then, concerning the solutions of this equation that belong to $L^{1}(\mathbb{R})$, the vector subspace $\mathbb{S} \cap L^{1}(\mathbb{R})$ of its $L^{1}(\mathbb{R})$ solutions, and the values of the dilation parameter $a$, we have the following

-If $|a|<1$ then the unique solution is the trivial one, $f=0$, a.e., and $\operatorname{dim}\left(\mathbb{S} \cap L^{1}(\mathbb{R})\right)=0$,

-If $|a|>1$ then there exist solutions in $\mathbb{Y}$ if and only if the infinite product $\prod_{n=0}^{\infty}\left(\hat{g}\left(\frac{\omega}{a^{n}}\right) /|a|\right)$ converges and is in $\mathcal{F}\left[L^{1}(\mathbb{R})\right] \backslash \mathbb{W}$. In this case we have $f=\left(\int_{\mathbb{R}} f(x) \mathrm{d} x\right) \mathcal{F}^{-1}\left(\prod_{n=0}^{\infty}\left(\hat{g}\left(\frac{\omega}{a^{n}}\right) /|a|\right)\right)$ and $\operatorname{dim}\left(\left(\left(\mathbb{S} \cap L^{1}(\mathbb{R})\right) \backslash \mathbb{W}\right) \cup\{0\}\right)=$ 1. On the other hand, if $\hat{f}(0)=0$ and $\prod_{n=0}^{\infty}\left(\hat{g}\left(\frac{\omega}{a^{n}}\right)|| a \mid\right) \in \mathcal{F}\left[L^{1}(\mathbb{R})\right] \backslash \mathbb{W}$ then the unique solution is the trivial one and $\operatorname{dim}\left(\mathbb{S} \cap L^{1}(\mathbb{R})\right)=0$,

-If $a=1(a=-1)$ then if $\hat{g} \neq 1$ a.e. $(\hat{g} . \hat{g}(-1 \cdot) \neq 1$ a.e.) then the unique solution is the trivial one and $\operatorname{dim}\left(\mathbb{S} \cap L^{1}(\mathbb{R})\right)=0$. However, there exist functions $g$ such that, both for $a=1$ or $a=-1, \mathbb{S} \cap L^{1}(\mathbb{R})$ is an infinite dimensional subspace of $L^{1}(\mathbb{R})$.

Proof. Propositions 1, 5, 8, 9, 11, 12, 13 and 14. For the only if part in the second statement, note that from proposition 5, taking $\omega=0$ we have $\left.\prod_{n=0}^{\infty}\left(\hat{g}\left(\frac{\omega}{a^{n}}\right) /|a|\right)\right|_{\omega=0}=\hat{f}(0) / \hat{f}(0)=1 \neq 0$ which implies $\prod_{n=0}^{\infty}\left(\hat{g}\left(\frac{\omega}{a^{n}}\right) /|a|\right) \notin$ W.

\section{Final Remarks}

Although the choice of dilation, or scale, parameter $a=1$ does not lead to a dilation integral equation, we remark that this choice leads to a singular Fredholm integral equation of the second type. A particular case of these equations, that corresponds to the choice $g(x)=\lambda e^{-|x|}, \lambda \in \mathbb{R}$, in equation (1), is the Lalesco-Picard equation, which is known to have solutions outside $L^{2}(\mathbb{R})$ but only the trivial solution in $L^{2}(\mathbb{R})$. See (Krasnov, Kiseliov and Makarenko, 1977). Since, in this case $\hat{g}(\omega)=\frac{2 \lambda}{1+\omega^{2}}$, by proposition (11), the Lalesco-Picard equation also has no other solution then the trivial one in $L^{1}(\mathbb{R})$. As we have shown, for convenient choices of $g$, these singular Fredholm integral equations present an infinitude of linearly independent solutions in $L^{1}(\mathbb{R})$, which is a very interesting feature. These equations have interest on their own and are the subject of another work.

\section{Acknowledgment}

The first author thanks NSSJC. 


\section{References}

Heil, C., \& Colella, D. (1994). Dilation Equations and the Smoothness of Compactly Supported Wavelets. In J. Benedetto and M. Frazier (EDS.), Wavelets: Mathematics and Applications, pp. 161-200. Boca Raton, FL: CRC Press.

Krasnov, M., Kiseliov, A., \& Makarenko, G. (1977). Integral Equations. (2nd ed.). Moscow: Mir Publishers, (Chapter 2).

Pinsky, M. A. (2009). Introduction to Fourier Analysis and Wavelets. Graduate Studies in Mathematics, vol. 102. Providence, Rhode Island: American Mathematical Society, (Chapter 2).

Rudin, W. (1980). Functional Analysis. (T. M. H. ed.). New Delhi: Tata McGraw-Hill Publishing Company LTD, (Chapter 7).

Strang, G. (1989). Wavelets and Dilation Equations: A Brief Introduction. SIAM Review, 31(4), 614-627. http://dx.doi.org/10.1137/1031128 